Internat. J. Math. \& Math. Sci.

Vol. 24, No. 10 (2000) 699-714

S0161171200002908

(C) Hindawi Publishing Corp.

\title{
STABLE FINITE ELEMENT METHODS FOR THE STOKES PROBLEM
}

\author{
YONGDEOK KIM and SUNGYUN LEE
}

(Received 11 March 1999)

\begin{abstract}
The mixed finite element scheme of the Stokes problem with pressure stabilization is analyzed for the cross-grid $P_{k}-P_{k-1}$ elements, $k \geq 1$, using discontinuous pressures. The $P_{k}^{+}-P_{k-1}$ elements are also analyzed. We prove the stability of the scheme using the macroelement technique. The order of convergence follows from the standard theory of mixed methods. The macroelement technique can also be applicable to the stability analysis for some higher order methods using continuous pressures such as Taylor-Hood methods, cross-grid methods, or iso-grid methods.
\end{abstract}

Keywords and phrases. Mixed finite element method, stabilization, Stokes problem.

2000 Mathematics Subject Classification. Primary 65N12, 65N15, 65N30.

1. Introduction. For the finite element approximation of the stationary Stokes equations several approaches appear in the literature $[6,9,13]$. The purpose of this paper is to analyze the mixed finite element scheme with pressure stabilization for some higher order triangular elements.

Let $\Omega$ be a bounded polygonal domain in $\mathbb{R}^{2}$. We consider the approximation of the stationary Stokes problem: find $\mathbf{u}=\left(u_{1}, u_{2}\right)$ and $p$ satisfying

$$
\begin{gathered}
-v \Delta \mathbf{u}+\nabla p=\mathbf{f} \quad \text { in } \Omega \\
\operatorname{div} \mathbf{u}=0 \quad \text { in } \Omega, \\
\mathbf{u}=\mathbf{0} \quad \text { on } \partial \Omega,
\end{gathered}
$$

where $\mathbf{u}$ is the fluid velocity, $p$ is the pressure, $\mathbf{f}$ is the given body force per unit mass, and $v>0$ is the viscosity. For the sake of simplicity, we take the viscosity equal to one. With the usual notation (see Section 2 for details) the standard variational formulation of this problem is: find $\mathbf{u} \in H_{0}^{1}(\Omega)^{2}$ and $p \in L_{0}^{2}(\Omega)$ such that

$$
\begin{gathered}
(\nabla \mathbf{u}, \nabla \mathbf{v})-(\operatorname{div} \mathbf{v}, p)=(\mathbf{f}, \mathbf{v}), \quad \mathbf{v} \in H_{0}^{1}(\Omega)^{2}, \\
(\operatorname{div} \mathbf{u}, q)=0, \quad q \in L_{0}^{2}(\Omega),
\end{gathered}
$$

where $(\cdot, \cdot)$ denote the usual $L^{2}$ inner products. For $\mathbf{f} \in H^{-1}(\Omega)^{2}$ this problem has a unique solution (cf. [9]). The standard mixed method based on (1.2) reads as follows: find $\mathbf{u}_{h} \in \mathbf{V}_{h} \subset H_{0}^{1}(\Omega)^{2}$ and $p \in P_{h} \subset L_{0}^{2}(\Omega)$ such that

$$
\begin{gathered}
\left(\nabla \mathbf{u}_{h}, \nabla \mathbf{v}\right)-\left(\operatorname{div} \mathbf{v}, p_{h}\right)=(\mathbf{f}, \mathbf{v}), \quad \mathbf{v} \in \mathbf{V}_{h}, \\
\left(\operatorname{div} \mathbf{u}_{h}, q\right)=0, \quad q \in P_{h} .
\end{gathered}
$$


Suppose that the finite element spaces $\mathbf{V}_{h}$ and $P_{h}$ indexed by the parameter $h, 0<h<1$, satisfy the inf-sup condition or the Babuška-Brezzi stability condition

$$
\inf _{0 \neq p \in P_{h}} \sup _{\mathbf{0} \neq \mathbf{v} \in \mathbf{v}_{h}} \frac{(\operatorname{div} \mathbf{v}, p)}{\|\mathbf{v}\|_{1}\|p\|_{0}} \geq C
$$

where $C$ is a positive constant independent of $h$. Then the theory of mixed methods states that the system (1.3) has a unique solution $\left(\mathbf{u}_{h}, p_{h}\right)$ satisfying

$$
\left\|\mathbf{u}-\mathbf{u}_{h}\right\|_{1}+\left\|p-p_{h}\right\|_{0} \leq C\left(\inf _{\mathbf{v} \in \mathbf{V}_{h}}\|\mathbf{u}-\mathbf{v}\|_{1}+\inf _{q \in P_{h}}\|p-q\|_{0}\right),
$$

where $(\mathbf{u}, p)$ is the solution to (1.2). Introducing the associated bounded bilinear form

$$
B(\mathbf{u}, p ; \mathbf{v}, q)=(\nabla \mathbf{u}, \nabla \mathbf{v})-(\operatorname{div} \mathbf{v}, p)+\epsilon(\operatorname{div} \mathbf{u}, q), \quad \epsilon= \pm 1,
$$

and the linear functional

$$
L(\mathbf{v}, q)=(\mathbf{f}, \mathbf{v}),
$$

we can recast the formulation (1.3) as follows: find $\left(\mathbf{u}_{h}, p_{h}\right) \in \mathbf{V}_{h} \times P_{h}$ such that

$$
B\left(\mathbf{u}_{h}, p_{h} ; \mathbf{v}, q\right)=L(\mathbf{v}, q), \quad(\mathbf{v}, q) \in \mathbf{V}_{h} \times P_{h} .
$$

Then the main result of $[1,2]$ says that $(1.5)$ holds provided

$$
\sup _{\mathbf{0} \neq(\mathbf{v}, q) \in \mathbf{V}_{h} \times P_{h}} \frac{B(\mathbf{u}, p ; \mathbf{v}, q)}{\|\mathbf{v}\|_{1}+\|q\|_{0}} \geq C\left(\|\mathbf{u}\|_{1}+\|p\|_{0}\right), \quad(\mathbf{u}, p) \in \mathbf{V}_{h} \times P_{h},
$$

where $C$ is a positive constant independent of $h$. Equation (1.9) will be referred to the stability condition for a bilinear form $B$ in general.

Since it could be a difficult task to verify (1.4) for a particular choice of velocity and pressure approximations, several methods have been developed aiming at stabilizing the discrete solution. We introduce an approximation scheme with pressure stabilization as follows: find $\left(\mathbf{u}_{h}, p_{h}\right) \in \mathbf{V}_{h} \times P_{h}$ such that

$$
B\left(\mathbf{u}_{h}, p_{h} ; \mathbf{v}, q\right)=L(\mathbf{v}, q), \quad(\mathbf{v}, q) \in \mathbf{V}_{h} \times P_{h}
$$

with

$$
\begin{gathered}
B(\mathbf{u}, p ; \mathbf{v}, q)=(\nabla \mathbf{u}, \nabla \mathbf{v})-(\operatorname{div} \mathbf{v}, p)+\epsilon(\operatorname{div} \mathbf{u}, q) \\
+\epsilon \beta \sum_{T \in \Gamma_{h}} h_{T}([[p]],[[q]])_{T}, \quad \epsilon= \pm 1, \\
L(\mathbf{v}, q)=(\mathbf{f}, \mathbf{v}) .
\end{gathered}
$$

Note that this method was considered as a special case of the stabilization procedures in $[7,10]$.

It is the purpose of this paper to show that the mixed finite element method (1.10) with pressure stabilization converges for some higher order elements using discontinuous pressures. More specifically, we establish the convergence of the cross-grid 
$P_{k}-P_{k-1}$ elements, $k \geq 1$ and the $P_{k}^{+}-P_{k-1}$ elements, $k \geq 2$ (see Section 3 ). To verify the stability condition (1.9), we will combine the ideas of macroelement technique in $[15,16,17]$ and the arguments in [8] for Galerkin least squares methods. Then the error estimate (1.5) follows in the usual manner. The macroelement technique can also be applicable to the stability analysis for some higher order methods using continuous pressures, such as Taylor-Hood methods, cross-grid methods, or iso-grid methods.

An outline of the paper is as follows. In Section 2, we develop the stability analysis and the macroelement technique together with the necessary preliminaries. The stability and convergence of various elements mentioned above are shown in Section 3 by an application of the results in Section 2 .

2. Macroelement technique and weak stability. Let $\mathscr{C}_{h}$ be a partitioning of $\bar{\Omega}$ into triangles for a bounded polygonal domain $\Omega \subset \mathbb{R}^{2}$. The triangulation is assumed to be regular in the usual sense, that is, for some $\sigma>1$,

$$
h_{K} \leq \sigma \rho_{K}, \quad K \in \mathscr{C}_{h},
$$

where $h_{K}$ is the diameter of element $K$ and $\rho_{K}$ is the diameter of the largest circle contained in $K$. The mesh parameter $h$ is given by $h=\max \left(h_{K}\right)$ and the set of all interelement boundaries will be denoted by $\Gamma_{h}$. We will not assume $\mathscr{C}_{h}$ to be quasiuniform.

The finite element subspaces of $P_{k}-P_{l}$ element are

$$
\begin{gathered}
\mathbf{V}_{h}=\left\{\mathbf{v}=\left(v_{1}, v_{2}\right) \in H_{0}^{1}(\Omega)^{2}:\left.v_{i}\right|_{K} \in P_{k}(K), i=1,2, K \in \mathscr{C}_{h}\right\}, \\
P_{h}=\left\{p \in L_{0}^{2}(\Omega):\left.p\right|_{K} \in P_{l}(K), K \in \mathscr{C}_{h}\right\},
\end{gathered}
$$

where $P_{s}$ denotes the collection of all polynomials of degree not greater than $s$ and $L_{0}^{2}(\Omega)$ denotes the subspace of $L^{2}(\Omega)$ of functions with zero mean value. Our notation is standard. The norms and seminorms in the Sobolev spaces $H^{1}(\Omega)^{2}$ are denoted by $\|\cdot\|_{1}$ and $|\cdot|_{1}$, respectively.

Given any regular triangulation $\mathscr{C}_{h}$, by a macroelement we now mean a connected set $M$ of adjoining elements $K$ from $\mathscr{C}_{h}$. Two macroelements $M$ and $\bar{M}$ are said to be equivalent if there is a continuous one-to-one and onto mapping $F: M \rightarrow \bar{M}$ such that $\left.F\right|_{K}$ is affine for each $K \subset M$. For a macroelement $M$ we define the spaces $\mathbf{V}_{0, M}$ and $P_{M}$ consistent with $\mathbf{V}_{h}$ and $P_{h}$ :

$$
\begin{aligned}
\mathbf{V}_{0, M} & =\left\{\mathbf{v} \in H_{0}^{1}(M)^{2}:\left.\mathbf{v}\right|_{K} \in P_{k}(K)^{2}, K \subset M\right\}, \\
P_{M} & =\left\{p \in L_{0}^{2}(M):\left.p\right|_{K} \in P_{l}(K), K \subset M\right\} .
\end{aligned}
$$

Further we define

$$
N_{M}=\left\{p \in P_{M}:\left(\mathbf{v}, \nabla_{h} p\right)_{M}=0, \mathbf{v} \in \mathbf{V}_{0, M}\right\},
$$

where $\nabla_{h} p$ is given by $\left.\nabla p\right|_{K}$ on each $K \subset M$. The collection of edges of elements in the interior of $M$ is denoted by $\Gamma_{M}$. The following seminorms defined in $P_{h}$ turns out to be very useful for the analysis below:

$$
|p|_{h}^{2}=\sum_{K \in \mathscr{C}_{h}} h_{K}^{2}\|\nabla p\|_{0, K}^{2}, \quad|[[p]]|_{h}^{2}=\sum_{T \in \Gamma_{h}} h_{T}([[p]],[[p]])_{T} .
$$


In $P_{M}$ we similarly define

$$
|p|_{M}^{2}=\sum_{K \subset M} h_{K}^{2}\|\nabla p\|_{0, K}^{2}, \quad|[[p]]|_{M}^{2}=\sum_{T \in \Gamma_{M}} h_{T}([[p]],[[p]])_{T} .
$$

Here, the collection of edges of elements in the interior of $M$ is denoted by $\Gamma_{M},\|\cdot\|_{0, K}$ is the $L^{2}$ norm on $K,(\cdot, \cdot)_{T}$ is the inner product in $L^{2}(T), h_{T}$ is the diameter of $T$, and $[[p]]_{T}$ is the jump in $p$ along $T$.

The macroelement technique is based on the macroelement partitioning $\mu_{h}$ satisfying the following conditions:

(M1) there is a fixed set of equivalence classes $\mathscr{D}_{i}, i=1, \ldots, q$, of macroelements such that each $M \in \mathcal{M}_{h}$ belongs to one of $\mathscr{D}_{i}$;

(M2) there is a positive integer $L$ such that each $K \in \mathscr{C}_{h}$ is contained in at least one and not more than $L$ macroelements of $\mu_{h}$;

(M3) each $M \in \mathscr{D}_{i}, i=1, \ldots, q$, satisfies

(M3a) $p \in N_{M}$ implies that $|p|_{M}=0$.

The usefulness of the macroelement concept and the above mesh-dependent norms is that it enables us to establish some weak stability estimates for the proof of (1.9).

REMARK 2.1. We have modified the presentation of Sternberg $[16,17]$ to deal with the pressure stabilization and discontinuous pressure approximations.

LEMMA 2.2. Let $\mathscr{D}$ be a class of equivalent macroelements. Suppose that (M3a) is valid for every $M \in \mathscr{D}$. Then there is a constant $C>0$ such that

$$
\sup _{\mathbf{0} \neq \mathbf{v} \in \mathbf{V}_{0, M}} \frac{\left(\mathbf{v}, \nabla_{h} p\right)_{M}}{|\mathbf{v}|_{1, M}} \geq C|p|_{M}, \quad p \in P_{M}
$$

holds for all $M \in \mathscr{D}$.

Proof. For $M \in \mathscr{D}$, define a scaling invariant

$$
\beta_{M}=\inf _{0 \neq p \in P_{M}} \sup _{\mathbf{0} \neq \mathbf{v} \in \mathbf{V}_{0, M}} \frac{\left(\mathbf{v}, \nabla_{h} p\right)_{M}}{|\mathbf{v}|_{1, M}|p|_{M}}
$$

which is positive from the hypothesis. By virtue of the argument of Sternberg (cf. $[15,17])$, the regularity condition (2.1) ensures that there is a constant $C$ such that $\beta_{M} \geq C>0$ for all $M \in \mathscr{D}$, which implies (2.8).

LEMMA 2.3. Suppose that there is a macroelement partitioning $\mu_{h}$ satisfying (M1), (M2), and (M3). Then the weak stability inequality

$$
\sup _{\mathbf{0} \neq \mathbf{v} \in \mathbf{V}_{h}} \frac{(\operatorname{div} \mathbf{v}, p)}{\|\mathbf{v}\|_{1}} \geq C_{1}|p|_{h}-C_{2}|[[p]]|_{h}, \quad p \in P_{h}
$$

is valid.

Proof. The local weak stability estimates (2.8) implies that for a given $p \in P_{h}$ and $M \in \mathcal{M}_{h}$, there is $\mathbf{v}_{M} \in \mathbf{V}_{h}$ with $\mathbf{v}_{M}=\mathbf{0}$ in $\Omega \backslash M$ such that

$$
\begin{gathered}
-\left(\mathbf{v}_{M}, \nabla_{h} p\right)_{M} \geq C|p|_{M}^{2}, \\
\left|\mathbf{v}_{M}\right|_{1}=\left|\mathbf{v}_{M}\right|_{1, M} \leq|p|_{M} .
\end{gathered}
$$


Then we have

$$
\begin{aligned}
& \left(\operatorname{div} \mathbf{v}_{M}, p\right)_{M}=-\left(\mathbf{v}_{M}, \nabla_{h} p\right)_{M}+\sum_{T \in \Gamma_{M}}\left(\mathbf{v}_{M} \cdot \mathbf{n},[[p]]\right)_{T}, \\
& \left(\operatorname{div} \mathbf{v}_{M}, p\right)_{M} \geq C|p|_{M}^{2}-\left(\sum_{T \in \Gamma_{M}} h_{T}^{-1}\left\|\mathbf{v}_{M}\right\|_{0, T}^{2}\right)^{1 / 2}|[[p]]|_{M} .
\end{aligned}
$$

Here $\mathbf{n}$ denotes a unit normal to $T$. From Lemma 2.4 we have the estimates

$$
\sum_{T \in \Gamma_{M}} h_{T}^{-1}\left\|\mathbf{v}_{M}\right\|_{0, T}^{2} \leq C \sum_{K \subset M} h_{K}^{-2}\left\|\mathbf{v}_{M}\right\|_{0, K}^{2} \leq C^{\prime}|\mathbf{v}|_{1, M}^{2} .
$$

Combining (2.12), (2.14), and (2.15), we obtain

$$
\left(\operatorname{div} \mathbf{v}_{M}, p\right)_{M} \geq C_{1}|p|_{M}^{2}-C_{2}|p|_{M}|[[p]]|_{M},
$$

where $C_{1}>0$ and $C_{2}>0$ can be taken independent of $M$. Next let us define $\mathbf{v} \in$ $\mathbf{V}_{h}$ through $\mathbf{v}=\sum_{M \in M_{h}} \mathbf{v}_{M}$. Then the macroelement conditions (M1), (M2), (M3), and (2.16) give

$$
\begin{aligned}
(\operatorname{div} \mathbf{v}, p) & =\sum_{M \in \mathcal{M}_{h}}\left(\operatorname{div} \mathbf{v}_{M}, p\right)_{M} \\
& \geq C_{1} \sum_{M \in \mathcal{M}_{h}}|p|_{M}^{2}-C_{2} \sum_{M \in \mathcal{M}_{h}}|p|_{M}|[[p]]|_{M} \\
& \geq C_{1}|p|_{h}^{2}-C_{2}\left(\sum_{M \in \mathcal{M}_{h}}|p|_{M}^{2}\right)^{1 / 2}\left(\sum_{M \in \mathcal{M}_{h}}|[[p]]|_{M}^{2}\right)^{1 / 2} \\
& \geq C_{1}|p|_{h}^{2}-C_{2}\left(\sqrt{L}|p|_{h}\right)\left(\sqrt{L}|[[p]]|_{h}\right) \\
& =C_{1}|p|_{h}^{2}-C_{2} L|p|_{h}|[[p]]|_{h} .
\end{aligned}
$$

Since

$$
\begin{aligned}
\|\mathbf{v}\|_{1} & \leq C|\mathbf{v}|_{1} \leq C \sum_{M \in \mathcal{M}_{h}}\left|\mathbf{v}_{M}\right|_{1, M} \leq C \sum_{M \in \mathcal{M}_{h}}|p|_{M} \\
& \leq C L \sum_{K \in \mathscr{C}_{h}} h_{K}\|\nabla p\|_{0, K} \leq C L|\Omega|^{1 / 2}|p|_{h},
\end{aligned}
$$

it follows from (2.17) that there are constants $C_{1}>0$ and $C_{2}>0$ satisfying (2.10). Here $|\Omega|$ denotes the measure of $\Omega$.

LEMMA 2.4. Let $M$ be a macroelement. Then we have for $\mathbf{u} \in \mathbf{V}_{h}$,

$$
\sum_{T \in \Gamma_{M}} h_{T}^{-1}\|\mathbf{u}\|_{0, T}^{2} \leq C \sum_{K \subset M} h_{K}^{-2}\|\mathbf{u}\|_{0, K}^{2},
$$

and for $\mathbf{u} \in \mathbf{V}_{0, M}$,

$$
\sum_{K \subset M} h_{K}^{-2}\|\mathbf{u}\|_{0, K}^{2} \leq C|\mathbf{u}|_{1, M}^{2},
$$

where constants $C>0$ depend only on the regularity constant $\sigma$ of (2.1). 
Proof. According to the argument of [3, page 1045] it is not difficult to see that for $\mathbf{u} \in \mathbf{V}_{h} 0$,

$$
\sum_{T \in \partial K} h_{T}^{-1}\|\mathbf{u}\|_{0, T}^{2} \leq C h_{K}^{-2}\|\mathbf{u}\|_{0, K}^{2}
$$

Then (2.19) follows immediately. Next, applying the argument of a proof of the inverse inequality for piecewise polynomials (cf. [13, page 195]), we can show that (2.20) holds for $\mathbf{u} \in \mathbf{V}_{0, M}$.

LEMmA 2.5. Suppose that either $k \geq 2$ in the definition (2.2) of $\mathbf{V}_{h}$ or $P_{h} \subset C(\Omega)$. Then there are two positive constants $C_{1}$ and $C_{2}$ such that

$$
\sup _{\mathbf{0} \neq \mathbf{v} \in \mathbf{V}_{h}} \frac{(\operatorname{div} \mathbf{v}, p)}{\|\mathbf{v}\|_{1}} \geq C_{1}\|p\|_{0}-C_{2}|p|_{h}, \quad p \in P_{h} .
$$

Proof. These are the cases (i) and (ii) of [8, Lemma 3.3]. See [8, pages 1685-1687] for the proof.

LEMMA 2.6. Under the assumption of Lemma 2.3 there are two positive constants $C_{1}$ and $C_{2}$ such that the weak stability inequality

$$
\sup _{\mathbf{0} \neq \mathbf{v} \in \mathbf{v}_{h}} \frac{(\operatorname{div} \mathbf{v}, p)}{\|\mathbf{v}\|_{1}} \geq C_{1}\|p\|_{0}-C_{2}|[[p]]|_{h}, \quad p \in P_{h}
$$

holds.

PROoF. Equation (2.23) follows from (2.10) and (2.22). To be more precise, let $C_{1}, C_{2}$ and $c_{1}, c_{2}$ be the constants in (2.10) and (2.22), respectively. For $0<t<1$ we have

$$
\begin{aligned}
\sup _{\mathbf{0} \neq \mathbf{v} \in \mathbf{V}_{h}} \frac{(\operatorname{div} \mathbf{v}, p)}{\|\mathbf{v}\|_{1}} & \geq(1-t)\left(C_{1}|p|_{h}-C_{2}|[[p]]|_{h}\right)+t\left(c_{1}\|p\|_{0}-c_{2}|p|_{h}\right) \\
& \geq t c_{1}\|p\|_{0}+\left((1-t) C_{1}-t c_{2}\right)|p|_{h}-(1-t) C_{2}|[[p]]|_{h} .
\end{aligned}
$$

Then (2.23) follows provided $t<C_{1}\left(C_{1}+c_{2}\right)^{-1}$.

We are ready to verify the stability condition (1.9) for the method (1.10). We do the case $\epsilon=1$. The other case $\epsilon=-1$ is similar.

THEOREM 2.7. Suppose that there is a macroelement partitioning $\mu_{h}$ satisfying (M1), (M2), and (M3) for a regular triangulation $\mu_{h}$ of $\Omega \subset \mathbb{R}^{2}$. Then given a stabilization parameter $\beta>0$ the stability condition (1.9) for the method (1.10) is valid.

Proof. Let $(\mathbf{u}, p) \in \mathbf{V}_{h} \times P_{h}$. First, we note that

$$
B(\mathbf{u}, p ; \mathbf{u}, p)=\|\nabla \mathbf{u}\|_{0}^{2}+\beta|[[p]]|_{h}^{2} \geq C_{1}\|\mathbf{u}\|_{1}^{2}+\beta|[[p]]|_{h}^{2} .
$$

Next, according to (2.23), there is $\mathbf{w} \in \mathbf{V}_{h}$ satisfying

$$
(\operatorname{div} \mathbf{w}, p) \geq C_{2}\|p\|_{0}^{2}-C_{3}\|p\|_{0}|[[p]]|_{h}
$$


and $\|\mathbf{w}\|_{1}=\|p\|_{0}$. Then for $t_{1}>0$ and $t_{2}>0$,

$$
\begin{aligned}
B(\mathbf{u}, p ;-\mathbf{w}, 0) & =-(\nabla \mathbf{u}, \nabla \mathbf{w})+(\operatorname{div} \mathbf{w}, p) \\
& \geq-\|\mathbf{u}\|_{1}\|p\|_{0}+C_{2}\|p\|_{0}^{2}-C_{3}\|p\|_{0}|[[p]]|_{h} \\
& \geq\left(C_{2}-\frac{t_{1}}{2}-\frac{C_{3} t_{2}}{2}\right)\|p\|_{0}^{2}-\frac{\|\mathbf{u}\|_{1}^{2}}{2 t_{1}}-\frac{C_{3}|[[p]]|_{h}^{2}}{2 t_{2}} .
\end{aligned}
$$

Choosing $t_{1}$ and $t_{2}$ small enough, we have

$$
B(\mathbf{u}, p ;-\mathbf{w}, 0) \geq C_{4}\|p\|_{0}^{2}-C_{5}\|\mathbf{u}\|_{1}^{2}-C_{6}|[[p]]|_{h}^{2}
$$

for some positive constants $C_{4}, C_{5}$, and $C_{6}$. Let us denote $(\mathbf{v}, q)=(\mathbf{u}-\delta \mathbf{w}, p)$. It follows from (2.25) and (2.28) that

$$
\begin{aligned}
B(\mathbf{u}, p ; \mathbf{v}, q) & =B(\mathbf{u}, p ; \mathbf{u}, p)+\delta B(\mathbf{u}, p ;-\mathbf{w}, 0) \\
& \geq \delta C_{4}\|p\|_{0}^{2}+\left(C_{1}-\delta C_{5}\right)\|\mathbf{u}\|_{1}^{2}+\left(\beta-\delta C_{6}\right)|[[p]]|_{h}^{2} .
\end{aligned}
$$

Choosing $0<\delta<\min \left\{C_{1} C_{5}^{-1}, \beta C_{6}^{-1}\right\}$ we obtain

$$
B(\mathbf{u}, p ; \mathbf{v}, q) \geq C_{7}\left(\|\mathbf{u}\|_{1}+\|p\|_{0}\right)^{2}
$$

for some positive constant $C_{7}$. On the other hand, we have

$$
\|\mathbf{v}\|_{1}+\|q\|_{0} \leq C_{8}\left(\|\mathbf{u}\|_{1}+\|p\|_{0}\right)
$$

for some positive constant $C_{8}$. Finally combining (2.30) and (2.31) we establish the stability condition (1.9) for the method (1.10).

The error estimates are now obtained in the usual manner from the stability inequality (1.9) and from the following estimates (cf. [8, 9]):

$$
\begin{gathered}
\left(\sum_{K \in \mathscr{C}_{h}} h_{K}^{2}\|\nabla q\|_{0, K}^{2}+\sum_{T \in \Gamma_{h}} h_{T}([[q]],[[q]])_{T}\right)^{1 / 2} \leq C\|q\|_{0}, \quad q \in P_{h}, \\
\inf _{q \in P_{h}}\left(\sum_{K \in \mathscr{C}_{h}} h_{K}^{2}\|\nabla(q-p)\|_{0, K}^{2}+\sum_{T \in \Gamma_{h}} h_{T}([[q-p]],[[q-p]])_{T}\right)^{1 / 2} \\
\leq C \inf _{q \in P_{h}}\|q-p\|_{0} \leq C h^{l+1}|p|_{l+1}, \quad p \in H^{l+1}(\Omega), \\
\inf _{\mathbf{v} \in \mathbf{V}_{h}}\|\mathbf{u}-\mathbf{v}\|_{1} \leq C h^{k}|\mathbf{u}|_{k+1}, \quad \mathbf{u} \in H^{k+1}(\Omega)^{2} .
\end{gathered}
$$

THEOREM 2.8. Let the assumptions of Theorem 2.7 be valid. Assume further that the solution $(\mathbf{u}, p)$ to (1.2) satisfies $\mathbf{u} \in H^{k+1}(\Omega)^{2}$ and $p \in H^{l+1}(\Omega)$. Then for $\beta>0$, (1.10) has a unique solution $\left(\mathbf{u}_{h}, p_{h}\right)$ satisfying (1.5) and

$$
\left\|\mathbf{u}-\mathbf{u}_{h}\right\|_{1}+\left\|p-p_{h}\right\|_{0} \leq C\left(h^{k}|\mathbf{u}|_{k+1}+h^{l+1}|p|_{l+1}\right) .
$$

If in addition $\Omega$ is a convex polygon, then we have

$$
\left\|\mathbf{u}-\mathbf{u}_{h}\right\|_{0} \leq C\left(h^{k+1}|\mathbf{u}|_{k+1}+h^{l+2}|p|_{l+1}\right) .
$$


Proof. We follow the argument of [8, page 1688]. Let $\tilde{\mathbf{u}} \in \mathbf{V}_{h}$ and $\tilde{p} \in P_{h}$ be the interpolants of $\mathbf{u}$ and $p$, respectively. The stability condition (1.9) of $B$ implies the existence of $(\mathbf{v}, q) \in \mathbf{V}_{h} \times P_{h}$ such that

$$
\begin{gathered}
\|\mathbf{v}\|_{1}+\|q\|_{0} \leq C, \\
\left\|\tilde{\mathbf{u}}-\mathbf{u}_{h}\right\|_{1}+\left\|\tilde{p}-p_{h}\right\|_{0} \leq B\left(\mathbf{u}_{h}-\tilde{\mathbf{u}}, p_{h}-\tilde{p} ; \mathbf{v}, q\right) .
\end{gathered}
$$

Since

$$
\begin{aligned}
B\left(\mathbf{u}_{h}-\tilde{\mathbf{u}}, p_{h}-\tilde{p} ; \mathbf{v}, q\right)= & B(\mathbf{u}-\tilde{\mathbf{u}}, p-\tilde{p} ; \mathbf{v}, q) \\
B(\mathbf{u}-\tilde{\mathbf{u}}, p-\tilde{p} ; \mathbf{v}, q) \leq & C\left(\|\mathbf{u}-\tilde{\mathbf{u}}\|_{1}^{2}+\|p-\tilde{p}\|_{0}^{2}+\sum_{T \in \Gamma_{h}} h_{T}([[p-\tilde{p}]],[[p-\tilde{p}]])_{T}\right)^{1 / 2} \\
& \cdot\left(\|\mathbf{v}\|_{1}^{2}+\|q\|_{0}^{2}+\sum_{T \in \Gamma_{h}} h_{T}([[q]],[[q]])_{T}\right)^{1 / 2}
\end{aligned}
$$

we get, from (2.32), (2.33), and (2.37),

$$
\left\|\tilde{\mathbf{u}}-\mathbf{u}_{h}\right\|_{1}+\left\|\tilde{p}-p_{h}\right\|_{0} \leq C\left(\|\mathbf{u}-\tilde{\mathbf{u}}\|_{1}+\|p-\tilde{p}\|_{0}\right),
$$

which gives (1.5) with the aid of the triangle inequality. Now (2.35) follows from (1.5), (2.33), and (2.34). Moreover, (2.36) follows from the Aubin-Nitsche argument using the a priori estimate [12],

$$
\|\mathbf{u}\|_{2}+\|p\|_{1} \leq C\|\mathbf{f}\|_{0}
$$

for a convex polygon.

3. Higher order stable elements. In this section, we apply, essentially, Theorems 2.7 and 2.8 for the analysis of several higher order stable elements. We will verify the macroelement conditions (M1), (M2), and (M3) and the approximation properties (2.33) and (2.34) for each method to establish the error estimates (2.35) and (2.36). Our main concern is the verification of the condition (M3), since a construction of macroelement partitioning satisfying (M1) and (M2) is not difficult and the approximation properties (2.33) and (2.34) follow from the standard interpolation theory. For the $P_{k}^{+}-P_{k-1}$ elements, $\mathbf{V}_{h}$ is enlarged using bubble functions on certain triangles. For the $P_{k}-P_{k}$ cross-grid elements or the $P_{k}-P_{k}$ iso-grid elements, the pressure triangulation $\mathscr{C}_{h}^{\prime}$ or $\tilde{\mathscr{C}}_{h}$ is coarser than the velocity triangulation $\mathscr{C}_{h}$. But the results of Section 2 can be interpreted without difficulty.

We begin by recalling that the barycentric coordinates $\lambda_{i}=\lambda_{i}(\mathbf{x}), 1 \leq i \leq 3$, of $\mathbf{x}=$ $(x, y) \in \mathbb{R}^{2}$ with respect to the points $A_{i}=\left(x_{i}, y_{i}\right), 1 \leq i \leq 3$, which makes a triangle $K$, are the unique solution of the linear system

$$
\sum_{i=1}^{3} \lambda_{i} A_{i}=\mathbf{x}, \quad \sum_{i=1}^{3} \lambda_{i}=1 .
$$




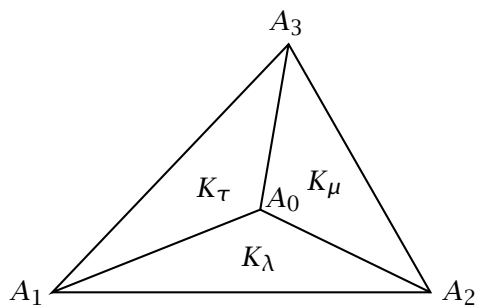

(a)

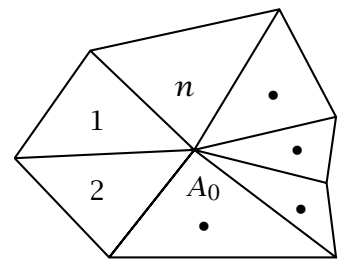

(b)

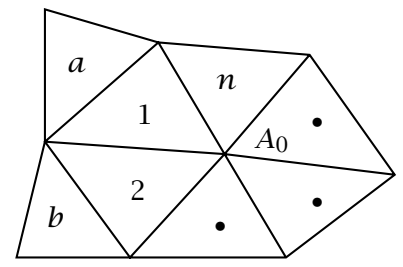

(c)

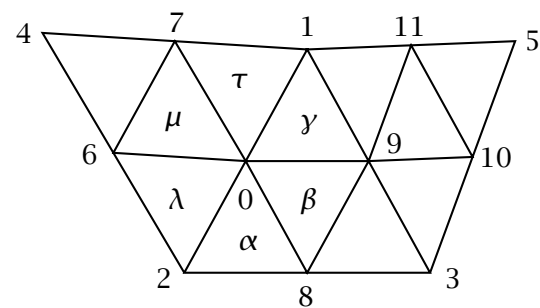

(d)

FIGURE 3.1. Examples of macroelement.

It follows that

$$
\left[\begin{array}{c}
\lambda_{1} \\
\lambda_{2}
\end{array}\right]=\frac{1}{\epsilon|K|}\left[\begin{array}{cc}
y_{2}-y_{3} & -\left(x_{2}-x_{3}\right) \\
-\left(y_{1}-y_{3}\right) & x_{1}-x_{3}
\end{array}\right]\left[\begin{array}{l}
x-x_{3} \\
y-y_{3}
\end{array}\right],
$$

where $|K|$ denotes the measure of $K$ and $\epsilon= \pm 1$ depending on the orientation of $A_{1}, A_{2}$, and $A_{3}$. Similar relations hold also for $\left[\begin{array}{l}\lambda_{1} \\ \lambda_{3}\end{array}\right]$ and $\left[\begin{array}{l}\lambda_{2} \\ \lambda_{3}\end{array}\right]$. Note that for any nonnegative integers $i, j, k$,

$$
\int_{K} \lambda_{1}^{i} \lambda_{2}^{j} \lambda_{3}^{k} d x=\frac{2 i ! j ! k !}{(i+j+k+2) !}|K|
$$

A few examples of macroelement are illustrated in Figure 3.1. For the macroelement in Figure 3.1a we interpret by $\lambda_{0}, \lambda_{1}, \lambda_{2}$ the barycentric coordinates on $K_{\lambda}$ with respect to $A_{0}, A_{1}$, and $A_{2}$. The similar interpretation of notation will apply for the other figures.

3.1. The cross-grid $P_{k}-P_{k-1}$ elements, $k \geq 1$, using discontinuous pressures. In the cross-grid methods using discontinuous pressures the triangulation $\mathscr{C}_{h}$ is obtained from a triangulation $\mathscr{C}_{h}^{\prime}$ by dividing each $K^{\prime} \in \mathscr{C}_{h}^{\prime}$ into three triangles inserting an interior vertex $A_{0}$ as in Figure $3.1 \mathrm{a}$, where $A_{0}$ is not necessarily the center of gravity of $K^{\prime}$. For $k \geq 1$ we define $\mathbf{V}_{h}$ by (2.2) and

$$
P_{h}=\left\{p \in L_{0}^{2}(\Omega):\left.p\right|_{K} \in P_{k-1}(K), K \in \mathscr{C}_{h}\right\} .
$$

LEMMA 3.1. Let $M$ be a macroelement consisting of three triangles aligned as in Figure 3.1a. Define $\mathbf{V}_{0, M}$ by (2.3) and

$$
P_{M}=\left\{p \in L_{0}^{2}(M):\left.p\right|_{K} \in P_{k-1}(K), K \subset M\right\} \quad \text { for } k \geq 2 .
$$

Then (M3a) is valid. 
Proof. Let $p \in N_{M}$ and write

$$
\left.p_{x}\right|_{K_{\lambda}}=\frac{g\left(\lambda_{0}, \lambda_{1}, \lambda_{2}\right)}{\left|K_{\lambda}\right|} .
$$

Suppose that $\left.p_{x}\right|_{K_{\mu}}=h\left(\mu_{0}, \mu_{3}, \mu_{2}\right) /\left|K_{\mu}\right|$. Here $\lambda_{i}$ 's and $\mu_{j}$ 's are the barycentric coordinates of $K_{\lambda}$ and $K_{\mu}$, respectively. Choose $\mathbf{u}=\left(u_{1}, 0\right) \in \mathbf{V}_{h, M}$ such that

$$
u_{1}= \begin{cases}\lambda_{0} \lambda_{2}(g+h)\left(\lambda_{0}, \lambda_{1}, \lambda_{2}\right) & \text { in } K_{\lambda}, \\ \mu_{0} \mu_{2}(g+h)\left(\mu_{0}, \mu_{3}, \mu_{2}\right) & \text { in } K_{\mu}, \\ 0 & \text { otherwise. }\end{cases}
$$

Then $\left(\mathbf{u}, \nabla_{h} p\right)_{M}=0$ gives an equation

$$
\left(\lambda_{0} \lambda_{2}(g+h)\left(\lambda_{0}, \lambda_{1}, \lambda_{2}\right), \frac{g\left(\lambda_{0}, \lambda_{1}, \lambda_{2}\right)}{\left|K_{\lambda}\right|}\right)_{K_{\lambda}}+\left(\mu_{0} \mu_{2}(g+h)\left(\mu_{0}, \mu_{3}, \mu_{2}\right), \frac{h\left(\mu_{0}, \mu_{3}, \mu_{2}\right)}{\left|K_{\mu}\right|}\right)_{K_{\mu}}=0 .
$$

On the other hand, it is not difficult to see from (3.3) that

$$
\frac{1}{K_{\lambda}} \int_{K_{\lambda}} \lambda_{0} \lambda_{2}\left(g^{2}+g h\right)\left(\lambda_{0}, \lambda_{1}, \lambda_{2}\right) d x=\frac{1}{K_{\mu}} \int_{K_{\mu}} \mu_{0} \mu_{2}\left(g^{2}+g h\right)\left(\mu_{0}, \mu_{3}, \mu_{2}\right) d x .
$$

Combining (3.8) and (3.9) we get

$$
\frac{1}{K_{\mu}} \int_{K_{\mu}} \mu_{0} \mu_{2}(g+h)^{2}\left(\mu_{0}, \mu_{3}, \mu_{2}\right) d x=0
$$

which implies

$$
(g+h)\left(\mu_{0}, \mu_{3}, \mu_{2}\right)=0
$$

Then we have

$$
\left.p_{x}\right|_{K_{\mu}}=-\frac{g\left(\mu_{0}, \mu_{3}, \mu_{2}\right)}{\left|K_{\mu}\right|} .
$$

By the same argument we obtain

$$
\left.p_{x}\right|_{K_{\tau}}=\frac{g\left(\tau_{0}, \tau_{3}, \tau_{1}\right)}{\left|K_{\tau}\right|},\left.\quad p_{x}\right|_{K_{\lambda}}=-\frac{g\left(\lambda_{0}, \lambda_{2}, \lambda_{1}\right)}{\left|K_{\lambda}\right|} .
$$

Thus $g$ is a polynomial satisfying

$$
\begin{aligned}
& g\left(\lambda_{0}, \lambda_{1}, \lambda_{2}\right)=-g\left(\lambda_{0}, \lambda_{2}, \lambda_{1}\right), \\
& g\left(\mu_{0}, \mu_{2}, \mu_{3}\right)=-g\left(\mu_{0}, \mu_{3}, \mu_{2}\right), \\
& g\left(\tau_{0}, \tau_{3}, \tau_{1}\right)=-g\left(\tau_{0}, \tau_{1}, \tau_{3}\right) .
\end{aligned}
$$

Let us consider the case $k=4$ first before we turn to the general case $k \geq 1$. From (3.6), (3.14), and the assumption $k=4$, we can write

$$
\left.p_{x}\right|_{K_{\lambda}}=\frac{a}{\left|K_{\lambda}\right|}\left(\lambda_{1}^{2}-\lambda_{2}^{2}\right)+\frac{b}{\left|K_{\lambda}\right|}\left(\lambda_{1} \lambda_{0}-\lambda_{2} \lambda_{0}\right)
$$


with two parameters $a$ and $b$ in $\mathbb{R}$. Similarly, $\left.p_{y}\right|_{K_{\lambda}}$ can be written as the right-hand side of (3.15) with the parameters $a$ and $b$ replaced by $a^{\prime}$ and $b^{\prime}$, respectively. Note also that $p_{x}$ and $p_{y}$ in $K_{\mu}$ or $K_{\tau}$ can be expressed using $a, b$ and $a^{\prime}, b^{\prime}$, respectively. Then from (3.6), (3.15), and

$$
\left(p_{x}\right)_{y}=\left(p_{y}\right)_{x}
$$

which are valid on each $K \in \mathscr{C}_{h}$, we get

$$
a\left(\lambda_{1}, y-\lambda_{2}, y\right)=a^{\prime}\left(\lambda_{1}, x-\lambda_{2}, x\right), \quad b\left(\lambda_{1}, y-\lambda_{2}, y\right)=b^{\prime}\left(\lambda_{1}, x-\lambda_{2}, x\right) .
$$

Applying (3.2) and (3.17) in $K_{\lambda}, K_{\mu}$, or $K_{\tau}$, we find that $\left(a, a^{\prime}\right)$ and $\left(b, b^{\prime}\right)$ satisfies the homogeneous system in $(s, t)$,

$$
s\left(x_{1}-x_{0}\right)+t\left(y_{1}-y_{0}\right)=0, \quad s\left(x_{2}-x_{0}\right)+t\left(y_{2}-y_{0}\right)=0,
$$

of which the solution is trivial since the determinant of the coefficient matrix is equal to $\left|K_{\lambda}\right| / 2>0$. This implies that $|p|_{M}=0$ when $k=4$. For the general case $k \geq 2$, we can write

$$
\left.p_{x}\right|_{K_{\lambda}}=\frac{1}{\left|K_{\lambda}\right|} \sum_{\substack{i+j+l=k-2 \\ i \geq j \geq 0, l \geq 0}} a_{i, j, l}\left(\lambda_{1}^{i} \lambda_{2}^{j}-\lambda_{2}^{i} \lambda_{1}^{j}\right) \lambda_{0}^{l} .
$$

Similarly, $\left.p_{y}\right|_{K_{\lambda}}$ can be written as the right-hand side of (3.19) with the parameters $a_{i, j, l}$ replaced by $a_{i, j, l}^{\prime}$. Moreover $p_{x}$ and $p_{y}$ in $K_{\mu}$ or $K_{\tau}$ can be expressed using $a_{i, j, l}$ and $a_{i, j, l}^{\prime}$ analogously. Then we find that $\left(a_{i, j, l}, a_{i, j, l}^{\prime}\right)$ satisfies (3.18) for each $i, j, l$. It follows that $a_{i, j, l}=a_{i, j, k, l}^{\prime}=0$ for each $i, j, l$ and that $\left.\nabla p\right|_{K}=0$, for all $K \subset M$. This completes the proof.

Thus we have a nonoverlapping macroelement partitioning, with one class of macroelements equivalent to $K^{\prime} \in \mathscr{C}_{h}^{\prime}$, which satisfies (M1), (M2), and (M3). A careful observation of the analysis of Section 2 also shows that for a nonoverlapping macroelement partitioning the coefficient of $\epsilon \beta$ in the approximation scheme (1.10) can be reduced to

$$
\sum_{K^{\prime} \in \mathscr{C}_{h}^{\prime}} \sum_{T \in \Gamma_{K^{\prime}}} h_{T}([[p]],[[q]])_{T},
$$

where $\Gamma_{K^{\prime}}$ denotes the collection of edges of elements of $\mathscr{C}_{h}$ in the interior of $K^{\prime}$. We will call the resulting scheme as the locally stabilized approximation scheme (cf. [11]). Since the approximation properties (2.33) and (2.34) are valid, we obtain the following result for the cross-grid $P_{k}-P_{k-1}$ elements, $k \geq 1$, using discontinuous pressures. 
THEOREM 3.2. Suppose that $\mathscr{C}_{h}$, which is obtained from $\mathscr{C}_{h}^{\prime}$, is a regular triangulation of $\Omega$. For $k \geq 1$, define $\mathbf{V}_{h}$ and $P_{h}$ by (2.2) and (3.4), respectively. Then Theorem 2.8 is valid with $\beta>0$ for the approximation scheme (1.10) or for the locally stabilized approximation scheme.

REMARK 3.3. The case $k=1$ can be considered as a special case of the scheme in [11].

3.2. The $P_{k}^{+}-P_{k-1}$ elements, $k \geq 2$, using discontinuous pressures. The argument of Lemma 3.1 shows that in general a macroelement $M$ of type (b) in Figure 3.1 consisting of $n$ triangles with a common vertex $A_{0}$ in the interior of $M$ satisfies (M3a) provided $n$ is odd. When the index $n$ of $A_{0}$ is even, we augment bubble functions on a triangle in $M$ in order to verify (M3a). For a regular triangulation $\mathscr{C}_{h}$ of $\Omega$, we can construct a macroelement partitioning $\mu_{h}$, consisting of macroelements of type (b) and (c) in Figure 3.1. Let $\mathscr{E}$ be a set of triangles in $\mathscr{C}_{h}$ such that for each macroelement $M \in \mathcal{M}_{h}$ with an interior vertex of even index there is a triangle $K \in \mathscr{E}$. Then we have the following result for $P_{k}^{+}-P_{k-1}$ element.

THEOREM 3.4. Suppose that $\mathscr{C}_{h}$ is a regular triangulation of $\Omega$. Define

$$
\begin{gathered}
\mathbf{V}_{h}=\left\{\mathbf{v} \in H_{0}^{1}(\Omega)^{2}:\left.\mathbf{v}\right|_{K} \in P_{k}(K)^{2}, K \in \mathscr{C}_{h} ;\left.\mathbf{v}\right|_{K} \in\left[P_{k}(K) \oplus \lambda_{1} \lambda_{2} \lambda_{3} P_{k-2}(K)\right]^{2}, K \in \mathscr{E}\right\}, \\
P_{h}=\left\{p \in L_{0}^{2}(\Omega):\left.p\right|_{K} \in P_{k-1}(K), K \in \mathscr{C}_{h}\right\} .
\end{gathered}
$$

Here $\lambda_{1}, \lambda_{2}, \lambda_{3}$ are the barycentric coordinates of the corresponding triangle $K$. Then Theorem 2.8 is valid for $k \geq 2$.

3.3. The cross-grid $P_{k}-P_{k}$ elements, $k \geq 1$. In the cross-grid $P_{k}-P_{k}$ elements, $k \geq 1$, the triangulation $\mathscr{C}_{h}$ for velocity is obtained from the triangulation $\tilde{\mathscr{C}}_{h}$ for pressure by dividing each $\tilde{K} \in \tilde{\mathscr{C}}_{h}$ into three triangles inserting an interior vertex $A_{0}$ as in Figure 3.1 a, where $A_{0}$ is not necessarily the center of gravity of $\tilde{K}$.

For $k \geq 1$ we define $\mathbf{V}_{h}$ by (2.2) and

$$
P_{h}=\left\{p \in L_{0}^{2}(\Omega):\left.p\right|_{\tilde{K}} \in P_{k}(\tilde{K}), \tilde{K} \in \tilde{\mathscr{C}}_{h}\right\} .
$$

LEMMA 3.5. Let $M$ be a macroelement consisting of three triangles aligned as in Figure 3.1a. Define $\mathbf{V}_{0, M}$ by (2.3) and

$$
P_{M}=\left\{p \in P_{k}(M)\right\} \quad \text { for } k \geq 1 .
$$

Then (M3a) is valid.

Proof. Let $p \in N_{M}$ and choose $\mathbf{u} \in \mathbf{V}_{0, M}$ such that

$$
\mathbf{u}= \begin{cases}\lambda_{0} \nabla p & \text { in } K_{\lambda}, \\ \mu_{0} \nabla p & \text { in } K_{\mu}, \\ \tau_{0} \nabla p & \text { in } K_{\tau} .\end{cases}
$$


Then $\left(\mathbf{u}, \nabla_{h} p\right)_{M}=0$ gives

$$
\left(\lambda_{0} \nabla p, \nabla p\right)_{K_{\lambda}}+\left(\mu_{0} \nabla p, \nabla p\right)_{K_{\mu}}+\left(\tau_{0} \nabla p, \nabla p\right)_{K_{\tau}}=0
$$

which implies $\left.\nabla p\right|_{K}=0$ for all $K \subset M$ and thus $|p|_{M}=0$.

Thus we have a nonoverlapping partitioning, with one class of macroelements equivalent to (a) in Figure 3.1, satisfying (M1), (M2), and (M3). Since the approximation properties (2.33) and (2.34) are valid, we obtain the following result for the cross-grid $P_{k}-P_{k}$ elements, $k \geq 1$.

THEOREM 3.6. Suppose that $\mathscr{C}_{h}$, which is obtained from $\tilde{\mathscr{C}}_{h}$, is a regular triangulation of $\Omega$. For $k \geq 1$, define $\mathbf{V}_{h}$ and $P_{h}$ by (2.2) and (3.22), respectively. Then Theorem 2.8 is valid with $\beta=0$.

3.4. The iso-grid $P_{k}-P_{k}$ elements, $k \geq 1$, using continuous pressures. In the $P_{k}-P_{k}$ iso-grid elements, $k \geq 1$, using continuous pressures, the triangulation $\mathscr{C}_{h}$ for velocity is obtained from a triangulation $\tilde{\mathscr{C}}_{h}$ for pressure by dividing each $\tilde{K} \in \tilde{\mathscr{C}_{h}}$ into four triangles inserting three vertices, one at each edge of $\tilde{K}$. Each of the inserted vertices is not necessarily a mid-point of the corresponding edge. For $k \geq 1$ we define $\mathbf{V}_{h}$ by (2.2) and

$$
P_{h}=\left\{p \in L_{0}^{2}(\Omega) \cap C(\Omega):\left.p\right|_{\tilde{K}} \in P_{k}(\tilde{K}), \tilde{K} \in \tilde{\mathscr{C}}_{h}\right\} .
$$

LEMMA 3.7. Let $M$ be a macroelement consisting of twelve triangles aligned as in Figure 3.1d. Define

$$
\begin{aligned}
\mathbf{V}_{0, M} & =\left\{\mathbf{v} \in H_{0}^{1}(M)^{2}:\left.\mathbf{v}\right|_{K} \in P_{k}(K)^{2}, K \in \mathscr{C}_{h} \cap M\right\}, \\
P_{M} & =\left\{p \in C(M):\left.p\right|_{\tilde{K}} \in P_{k}(\tilde{K}), \tilde{K} \in \tilde{\mathscr{C}}_{h} \cap M\right\} \quad \text { for } k \geq 1 .
\end{aligned}
$$

Then (M3a) is valid.

Proof. Let $p \in N_{M}$ and define

$$
g= \begin{cases}\tau_{0} & \text { in } K_{017}, \\ \mu_{0} & \text { in } K_{076}, \\ \lambda_{0} & \text { in } K_{062}, \\ \alpha_{0} & \text { in } K_{028}, \\ \beta_{0} & \text { in } K_{089}, \\ \gamma_{0} & \text { in } K_{091}, \\ 0 & \text { otherwise }\end{cases}
$$

where $K_{i j k}$ denotes the triangle with vertices $A_{i}, A_{j}$, and $A_{k}$. Let $\mathbf{e}=\left(e_{1}, e_{2}\right)$ be the unit vector in the direction of $\overrightarrow{A_{1} A_{2}}$, and choose $\mathbf{u}=\left(e_{1} g p_{\mathbf{e}}, e_{2} g p_{\mathbf{e}}\right) \in \mathbf{V}_{0, M}$. Note that $p_{\mathbf{e}}=\nabla p \cdot \mathbf{e}$ is continuous on $K_{123} \cup K_{142}$. Then $\left(\mathbf{u}, \nabla_{h} p\right)_{M}=0$ gives

$$
\left(\tau_{0} p_{\mathrm{e}}, p_{\mathrm{e}}\right)_{K_{017}}+\left(\mu_{0} p_{\mathrm{e}}, p_{\mathrm{e}}\right)_{K_{076}}+\cdots+\left(\gamma_{0} p_{\mathrm{e}}, p_{\mathrm{e}}\right)_{K_{091}}=0
$$


which implies $\left.p_{\mathbf{e}}\right|_{K_{017}}=0, \ldots,\left.p_{\mathbf{e}}\right|_{K_{091}}=0$. The similar argument gives also $\left.p_{\mathbf{d}}\right|_{K_{091}}=0$, where $\mathbf{d}$ is the unit vector in the direction of $\overrightarrow{A_{2} A_{4}}$. Since $\mathbf{e}$ and $\mathbf{d}$ are linearly independent, we get $\left.\nabla p\right|_{K_{091}}=0$ and so $\left.\nabla p\right|_{K_{123}}=0$. Next consider $\mathbf{u}=\left(e_{2} g p_{\mathbf{e}}^{\perp},-e_{1} g p_{\mathbf{e}}^{\perp}\right) \in$ $\mathbf{V}_{0, M}$, where $p_{\mathrm{e}}^{\perp} \in P_{k-1}\left(\mathbb{R}^{2}\right)$ is the extension of $\left.\left(e_{2} p_{x}-e_{1} p_{y}\right)\right|_{K_{142}}$. Then $\left(\mathbf{u}, \nabla_{h} p\right)_{M}=0$ gives

$$
\left(\tau_{0} p_{\mathrm{e}}^{\perp}, p_{\mathrm{e}}^{\perp}\right)_{K_{017}}+\left(\mu_{0} p_{\mathrm{e}}^{\perp}, p_{\mathrm{e}}^{\perp}\right)_{K_{076}}+\left(\lambda_{0} p_{\mathrm{e}}^{\perp}, p_{\mathrm{e}}^{\perp}\right)_{K_{062}}=0
$$

which implies $\left.p_{\mathbf{e}}^{\perp}\right|_{K_{062}}=0$. Since $\left(e_{1}, e_{2}\right)$ and $\left(e_{2},-e_{1}\right)$ are orthogonal, $\left.p_{\mathbf{e}}\right|_{K_{062}}=\left.p_{\mathbf{e}}^{\perp}\right|_{K_{062}}$ $=0$ implies $\left.\nabla p\right|_{K_{062}}=0$ and so $\left.\nabla p\right|_{K_{142}}=0$. The analogous argument also gives $\left.\nabla p\right|_{K_{135}}=0$. It follows that $|p|_{M}=0$.

Now it is easy to construct a macroelement partitioning, with only one class of macroelements equivalent to (d) in Figure 3.1, satisfying (M1), (M2), and (M3). Since the approximation properties (2.33) and (2.34) are valid, we have the following result for the iso-grid $P_{k}-P_{k}$ elements, $k \geq 1$, using continuous pressures.

THEOREM 3.8. Suppose that $\mathscr{C}_{h}$, which is obtained from $\tilde{\mathscr{C}}_{h}$, is a regular triangulation of $\Omega$. For $k \geq 1$, define $\mathbf{V}_{h}$ and $P_{h}$ by (2.2) and (3.26), respectively. Then Theorem 2.8 is valid with $\beta=0$.

3.5. The $P_{k}-P_{k-1}$ Taylor-Hood elements, $k \geq 2$

LEMMA 3.9. Let $M$ be a macroelement consisting of three triangles aligned as in Figure 3.1a. Define $\mathbf{V}_{0, M}$ by (2.3) and

$$
P_{M}=\left\{p \in C(M):\left.p\right|_{K} \in P_{k-1}(K), K \subset M\right\} \quad \text { for } k \geq 2 .
$$

Then (M3a) is valid.

Proof. Let $\mathbf{e}=\left(e_{1}, e_{2}\right)$ be the unit vector in the direction of $\overrightarrow{A_{0} A_{2}}$. Choose $\mathbf{u}=$ $\left(u_{1}, u_{2}\right) \in \mathbf{V}_{0, M}$ such that

$$
u_{1}=\left\{\begin{array}{ll}
e_{1} \lambda_{0} \lambda_{2} p_{\mathrm{e}} & \text { in } K_{\lambda}, \\
e_{1} \mu_{0} \mu_{2} p_{\mathrm{e}} & \text { in } K_{\mu}, \\
0 & \text { in } K_{\tau},
\end{array} \quad u_{2}= \begin{cases}e_{2} \lambda_{0} \lambda_{2} p_{\mathrm{e}} & \text { in } K_{\lambda}, \\
e_{2} \mu_{0} \mu_{2} p_{\mathrm{e}} & \text { in } K_{\mu}, \\
0 & \text { in } K_{\tau},\end{cases}\right.
$$

where $p_{\mathbf{e}}=\nabla p \cdot \mathbf{e}$. Let $p \in N_{M}$. Then $\left(\mathbf{u}, \nabla_{h} p\right)_{M}=0$ gives

$$
\left(\lambda_{0} \lambda_{2} p_{\mathbf{e}}, p_{\mathbf{e}}\right)_{K_{\lambda}}+\left(\mu_{0} \mu_{2} p_{\mathbf{e}}, p_{\mathbf{e}}\right)_{K_{\mu}}=0
$$

which implies $\left.p_{\mathbf{e}}\right|_{K_{\lambda}}=0$ and $\left.p_{\mathbf{e}}\right|_{K_{\mu}}=0$. Similarly, we have $\left.p_{\mathbf{d}}\right|_{K_{\lambda}}=0$ and $\left.p_{\mathbf{d}}\right|_{K_{3}}=0$, where $\mathbf{d}$ is the unit vector in the direction of $\overrightarrow{A_{0} A_{3}}$. Since $\mathbf{e}$ and $\mathbf{d}$ are linearly independent, we have $\nabla p=0$ in $K_{\mu}$. By the same reasoning we also have $\nabla p=0$ in $K_{\lambda}$ or $K_{\tau}$. It follows that $|p|_{M}=0$.

Now it is not difficult to construct a macroelement partitioning $M_{h}$, consisting of macroelements of types (b) and (c) in Figure 3.1, satisfying (M1), (M2), and (M3). Since the approximation properties (2.33) and (2.34) are valid, we get the following result for $P_{k}-P_{k-1}$ Taylor-Hood elements, $k \geq 2$. 
THEOREM 3.10. Suppose that $\mathscr{C}_{h}$ is a regular triangulation of $\Omega$. For $k \geq 2$, define $\mathbf{V}_{h}$ by (2.2) and

$$
P_{h}=\left\{p \in L_{0}^{2}(\Omega) \cap C(\Omega):\left.p\right|_{K} \in P_{k-1}(K), K \in \mathscr{C}_{h}\right\} .
$$

Then Theorem 2.8 is valid with $\beta=0$.

REMARK 3.11. It should be pointed out that some restrictions (cf. [5]) inherited from the result of Scott and Vogelius [14] are removed here. See [4] for a different proof.

ACKNOWLEDGEMENT. This work was supported in part by KAIST.

\section{REFERENCES}

[1] I. Babuška, Error-bounds for finite element method, Numer. Math. 16 (1970/1971), $322-$ 333. MR 44\#6166. Zbl 214.42001.

[2] I. Babuška and A. K. Aziz, Survey lectures on the mathematical foundations of the finite element method, The Mathematical Foundations of the Finite Element Method with Applications to Partial Differential Equations (Proc. Sympos., Univ. Maryland, Baltimore, Md., 1972) (New York), Academic Press, 1972, With the collaboration of G. Fix and R. B. Kellogg, pp. 1-359. MR 54\#9111. Zbl 268.65052.

[3] I. Babuška, J. Osborn, and J. Pitkäranta, Analysis of mixed methods using mesh dependent norms, Math. Comp. 35 (1980), no. 152, 1039-1062. MR 81m:65166. Zbl 472.65083.

[4] D. Boffi, Stability of higher order triangular Hood-Taylor methods for the stationary Stokes equations, Math. Models Methods Appl. Sci. 4 (1994), no. 2, 223-235. MR 95h:65079. Zbl 804.76051.

[5] F. Brezzi and R. S. Falk, Stability of higher-order Hood-Taylor methods, SIAM J. Numer. Anal. 28 (1991), no. 3, 581-590. MR 91m:65272. Zbl 731.76042.

[6] F. Brezzi and M. Fortin, Mixed and Hybrid Finite Element Methods, Springer Series in Computational Mathematics, vol. 15, Springer-Verlag, New York, 1991. MR 92d:65187. Zbl 788.73002.

[7] J. Douglas, Jr. and J. P. Wang, An absolutely stabilized finite element method for the Stokes problem, Math. Comp. 52 (1989), no. 186, 495-508. MR 89j:65069. Zbl 669.76051.

[8] L. P. Franca and R. Stenberg, Error analysis of Galerkin least squares methods for the elasticity equations, SIAM J. Numer. Anal. 28 (1991), no. 6, 1680-1697. MR 92k:73066. Zbl 759.73055.

[9] V. Girault and P.-A. Raviart, Finite Element Methods for Navier-Stokes Equations, Theory and Algorithms, Springer Series in Computational Mathematics, vol. 5, SpringerVerlag, Berlin, New York, 1986. MR 88b:65129. Zbl 585.65077.

[10] T. J. R. Hughes and L. P. Franca, A new finite element formulation for computational fluid dynamics. VII. The Stokes problem with various well-posed boundary conditions: symmetric formulations that converge for all velocity/pressure spaces, Comput. Methods Appl. Mech. Engrg. 65 (1987), no. 1, 85-96. MR 89j:76015g. Zbl 635.76067.

[11] N. Kechkar and D. Silvester, Analysis of locally stabilized mixed finite element methods for the Stokes problem, Math. Comp. 58 (1992), no. 197, 1-10. MR 92e:65138. Zbl 738.76040 .

[12] R. B. Kellogg and J. E. Osborn, A regularity result for the Stokes problem in a convex polygon, J. Functional Analysis 21 (1976), no. 4, 397-431. MR 53\#8649. Zbl 317.35037.

[13] A. Quarteroni and A. Valli, Numerical Approximation of Partial Differential Equations, Springer Series in Computational Mathematics, 23, Springer-Verlag, Berlin, 1994. MR 95i:65005. Zbl 803.65088. 
[14] L. R. Scott and M. Vogelius, Norm estimates for a maximal right inverse of the divergence operator in spaces of piecewise polynomials, RAIRO Modél. Math. Anal. Numér. 19 (1985), no. 1, 111-143. MR 87i:65190. Zbl 608.65013.

[15] R. Stenberg, Analysis of mixed finite elements methods for the Stokes problem: a unified approach, Math. Comp. 42 (1984), no. 165, 9-23. MR 84k:76014. Zbl 535.76037.

[16] _ Error analysis of some finite element methods for the Stokes problem, Math. Comp. 54 (1990), no. 190, 495-508. MR 90h:65189. Zbl 702.65095.

[17] _ A technique for analysing finite element methods for viscous incompressible flow, Internat. J. Numer. Methods Fluids 11 (1990), no. 6, 935-948, The Seventh International Conference on Finite Elements in Flow Problems (Huntsville, AL, 1989). MR 91k:76112. Zbl 704.76017.

YONGDEOK KIM: DEPARTMENT OF MATHEMATICS, KAIST, TAEJON, 305-701, KOREA

E-mail address: ydkimjia@hananet.net, ydkim@mathx.kaist.ac.kr

SUnGyun LeE: DePARTMENT OF MATHEMATICs, KAIST, TAEJON, 305-701, KoREA

E-mail address: sylee@mathx.kaist.ac.kr 


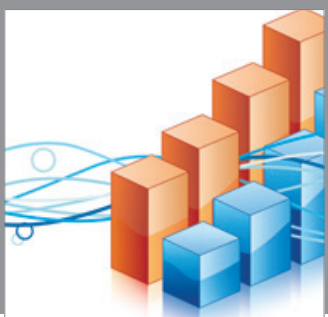

Advances in

Operations Research

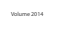

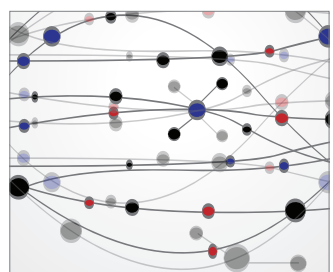

\section{The Scientific} World Journal
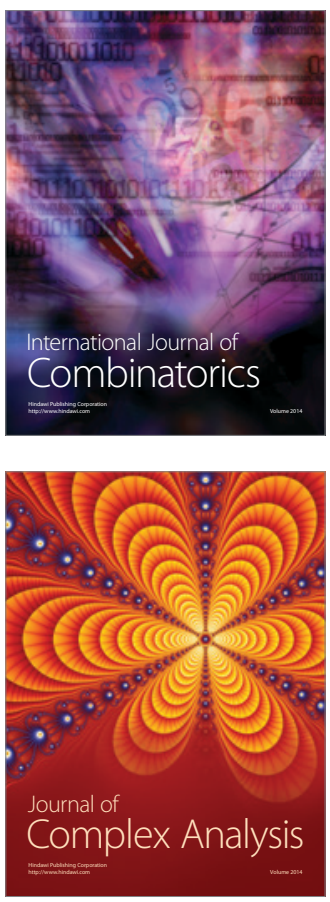

International Journal of

Mathematics and

Mathematical

Sciences
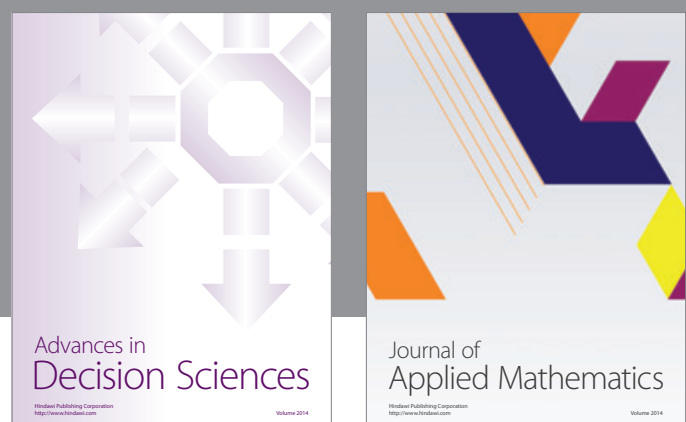

Journal of

Applied Mathematics
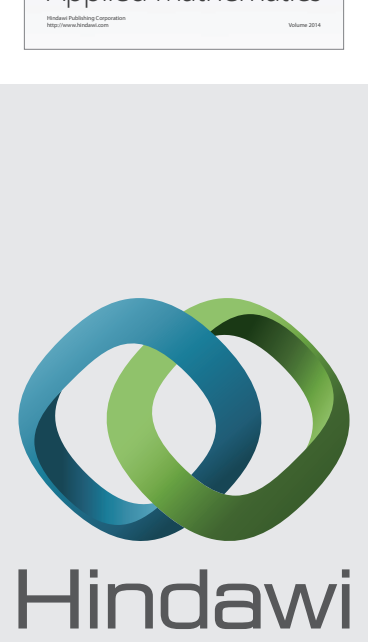

Submit your manuscripts at http://www.hindawi.com
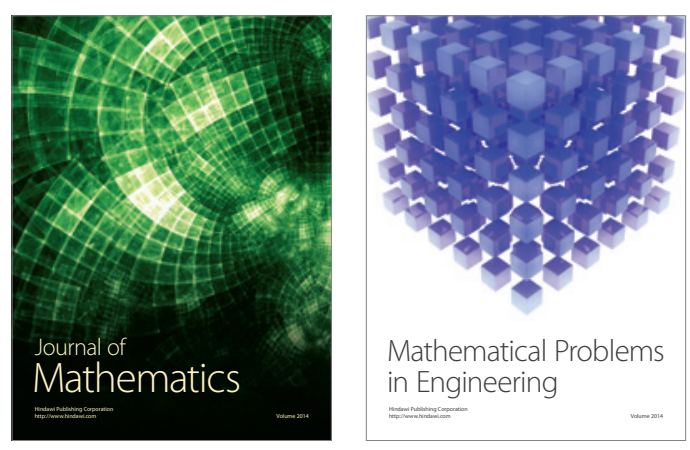

Mathematical Problems in Engineering
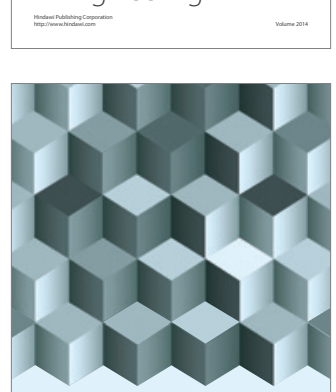

Journal of

Function Spaces
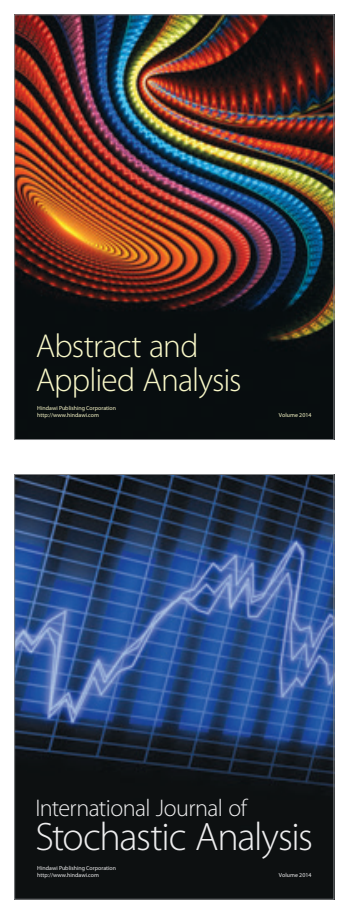

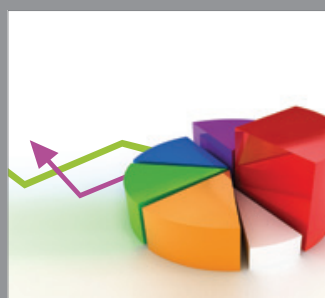

ournal of

Probability and Statistics

Promensencen
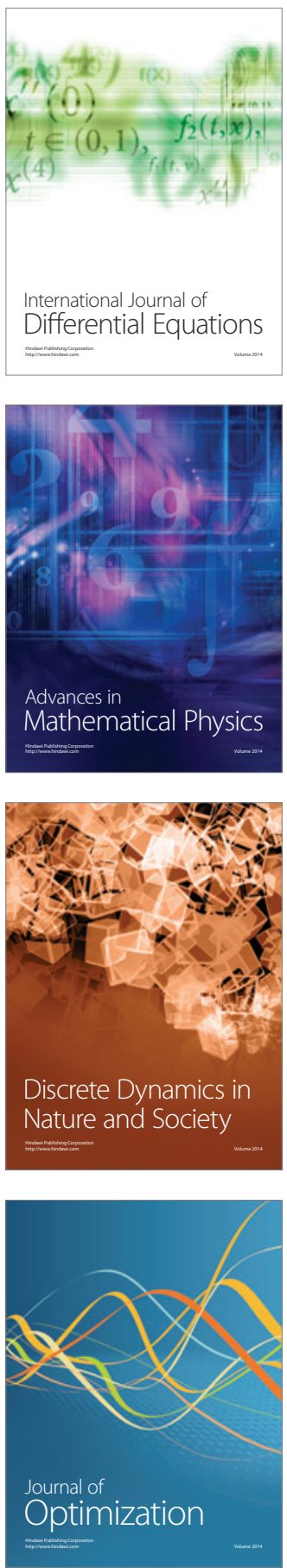